\title{
A MAS Framework for the Negotiation based Operational Integration of Virtual Enterprises
}

\author{
G. Wang, T. N. Wong, X. H. Wang \\ Department of Industrial and Manufacturing Systems Engineering \\ The University of Hong Kong \\ Hong Kong \\ wanggong@hkusua.hku.hk, tnwong@hku.hk,wangxh@hkusua.hku.hk
}

\begin{abstract}
The virtual enterprise (VE) is an effective way of business operation in the dynamic global market. This paper proposes a multi-agent system (MAS) framework for the negotiation based operational integration of VEs. Firstly, integrating the major VE operational activities into agent functions, a MAS framework is developed to represent the whole life cycle of VEs. Secondly, a negotiation protocol supporting agent argumentation is designed to handle the partner selection and conflict solving negotiation scenarios in the VE context. Finally, the proposed MAS framework is implemented upon the JADE platform. Simulation experiments have been carried out to verify the agent interaction sequences in the negotiation protocol.
\end{abstract}

Keywords-virtual enterprise; multi-agent system; negotiation; protocol; simulation

\section{INTRODUCTION}

The virtual enterprise (VE) is a way of organizing manufacturing and supply chain activities, where different and independent partners exploit business opportunities by establishing an enterprise cooperative [1]. It is a temporary and flexible form of supply chain strategic alliances in the dynamic global market. Throughout the whole VE life span, geographically distributed VE participants have to negotiate and cooperate to achieve mutual gains as well as ultimate customer satisfaction. Therefore, a VE can be portrayed as a distributed system comprising of autonomous and cooperative sub-systems.

Towards the distributed, autonomous and cooperative features, the multi-agent system (MAS) technology is suitable to model the VE system. The VE can be mapped into the MAS where autonomous intelligent agents coordinate to solve distributed problems. Integrating the major VE operational activities into agent functions, the MAS-based VE architecture can both facilitate the operational integration of VEs and enhance the business automation and intelligence.

In the context of VEs, negotiation is a prevalent form of interaction when $\mathrm{VE}$ partner entities encountering to solve transaction or scheduling conflicts. To adapt to this specific application requirement, agents in the MAS-based VEs should be equipped with sophisticated automated negotiation abilities. Generally, the automated negotiation model should be capable of dealing with the multi-issue multi-lateral negotiation which is a common scenario of transaction in VEs, meanwhile, agents are supposed to argue about their current status and other mental attitudes during the negotiation process.

An integrated operational system for VEs will facilitate the fluent control of transaction, scheduling and manufacturing procedures within VEs. However, the existing MAS architectures are more or less confined to static architectures representing the infrastructure of VE networking or only partial vision of the whole VE operational procedures [2, 3]. To better describe the operational procedures and the dynamic negotiation function flows of VEs, the main objective of this paper is to propose a successive MAS architecture integrating the major VE organizational procedures assisted by an agent negotiation protocol which can support multi-issue multi-lateral negotiation and agent argumentation in the VE context. The paper will be structured as follows. In section 2, a MAS-based VE architecture will be set up to integrate major functions in the whole VE life cycle. Section 3 will propose the agent negotiation protocol for partner selection and conflict solving negotiations. Section 4 will show the system implementation and experimental results. Finally, in section 5, conclusions will be drawn, with suggestions for future research.

\section{THE MAS ARCHITECTURE FOR VES}

To integrate the major activities of the VE, the four-phase VE life cycle concept $[4,5]$ has been adopted in the proposed MAS architecture, including identification, formation, operation and dissolution phases.

In the identification phase, the business opportunities are identified through market analysis of the VE initiator. In the formation phase, qualified VE partners will be selected from potential VE partners. In the operation phase, VE partners will perform the assigned contractual responsibilities. The VE initiator coordinates the VE participants' processes to finally identify the business opportunities. In the dissolution phase, the $\mathrm{VE}$ is to be dissolved after all the transaction processes of products and services have been accomplished.

The aforementioned VE functioning activities are integrated into six types of agents in the MAS, which are task decomposer agent (TDA), coordinator agent (CA), knowledge manager agent (KMA), buyer agent (BA), seller agent (SA), and performance evaluator agent (PEA). Sine the VE initiator takes the leading role in the VE, it comprises all the six types of 
agents. VE partners are simplified to be a buyer or seller agent. Figure 1 shows the agent system architecture. The whole system is initialized by some market opportunity, and then agents work cooperatively to deliver this opportunity. In the
VE operation phase, performance data of VE partners will be input into the system to evaluate partners' performances. Agent functions are generally described as follows.

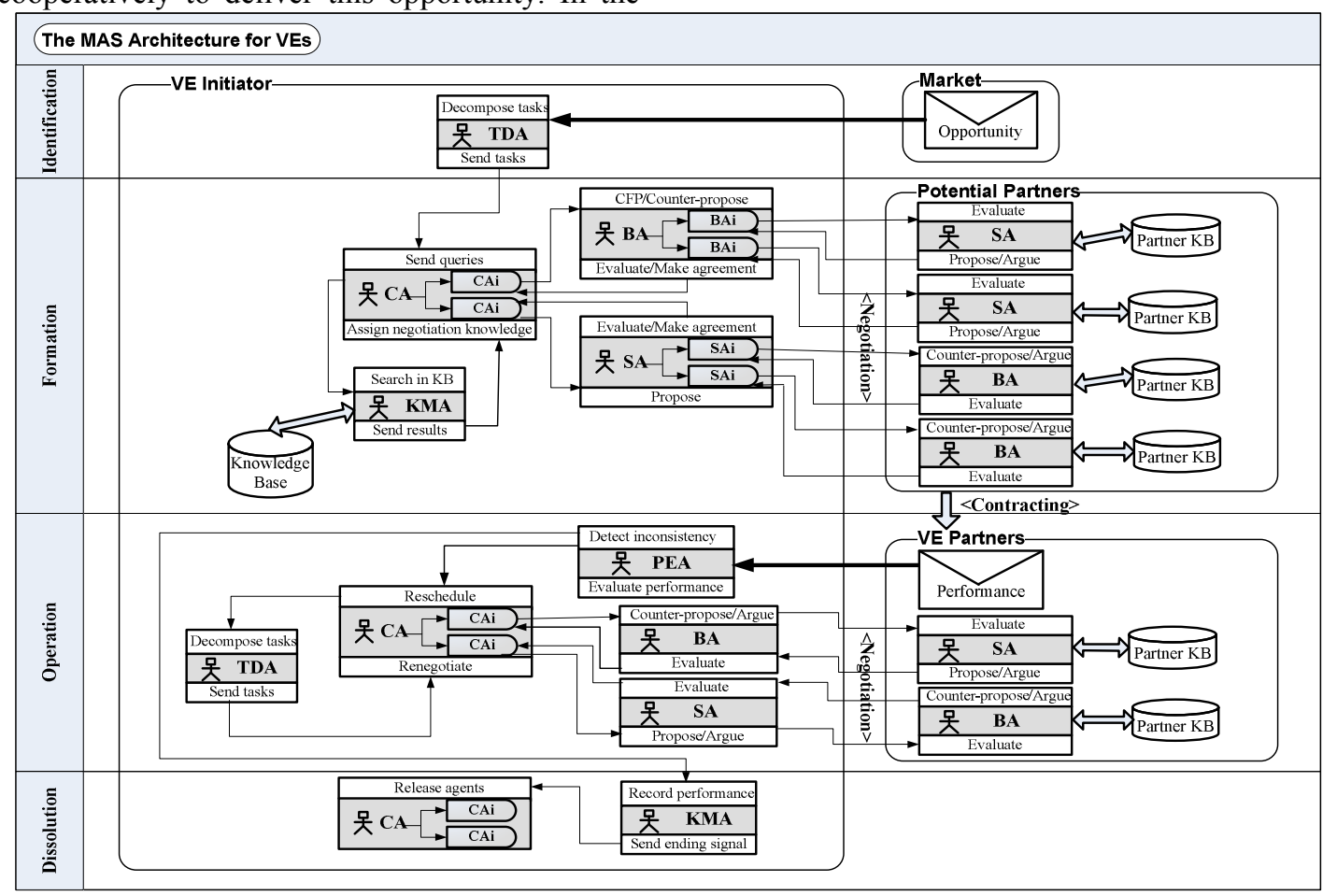

Figure 1. The general MAS architecture for the operational integration of VEs

- The task decomposer agent (TDA) is responsible for decomposing market opportunities and scheduling plans into subsidiary single tasks and sending the tasks to the coordinator agent for negotiation.

- The coordinator agent (CA) takes the role of process organizer throughout the whole $\mathrm{VE}$ life cycle. A separate coordinator agent instance (CAi) is in charge of a single task. In the VE formation phase, it delivers knowledge queries to the knowledge manager agent and initializes the buyer or seller agent to carry out negotiations according to the related negotiation knowledge. In the operation phase, the CA will reschedule the production process and renegotiate with the VE partners if inconsistencies are detected from the partners' performances. In the dissolution phase, it will release all the agents when receiving the ending signal.

- $\quad$ The knowledge manager agent (KMA) is designed for handling the knowledge related activities, including knowledge recording, classifying and retrieving. Knowledge governed by the KMA is supposed to be organized in the form of ontology. When the knowledge queries are received from the CA, the KMA will retrieve the knowledge within the knowledge base. In the VE dissolution phase, it will record all the performance information and send the ending signal.

- The buyer agent (BA) and seller agent (SA) are negotiation counterparts which have the same functionality. They perform negotiation procedures for the benefit of trading opponents just as the buyer and seller bargaining in the real world. If the VE formation requirement involves procurement or contract manufacturing type of tasks, the CA will initialize a BA. Conversely, the SA will be initialized when the requirement involves distribution tasks (e.g. supplying products to distributors). Negotiations are carried out between each pair of BA instance (BAi) and SA or SA instance (SAi) and BA, until the mutual agreement is achieved. In the VE formation phase, final negotiation outcomes will be sent back to the CA for selecting the bidding winners from the potential VE partners. The bidding winners will be contracted as VE partners. In the operation phase, the BA and SA can still negotiate if there are inconsistencies in the manufacturing and delivery processes between VE initiator and partners.

- The performance evaluator agent (PEA) is responsible for evaluating VE partners' performances when the performance data are collected in the VE operation phase. If inconsistencies are detected from the partners' performances, the PEA will inform the CA about the current situation.

\section{Agent Negotiation Protocol}

In the proposed MAS framework for VEs, agents can solve $\mathrm{VE}$ formation and operation problems through automated negotiation. The agents will perform the negotiation activities 
in behalves of the VE members after their desires and functions are set by human users. Generally, there are three broad areas needed to be considered when building a sophisticated agent negotiation model [6]: negotiation issues, negotiation protocol and negotiation strategies. The negotiation protocol regulates the encounter rules between agents. The contract net protocol (CNP) [7] is a commonly used negotiation protocol in bilateral negotiation models. CNP defines the basic inviting, bidding and awarding functions which may be involved in negotiation procedures. Based on the CNP, the negotiation protocol in the proposed MAS framework will be designed to facilitate both the one-to-one and one-to-many negotiation scenarios. Meanwhile, encounter rules and message structures will be presented in the protocol to regulate the agent argumentation.

\section{A. The Negotiation Protocol}

The proposed negotiation protocol for VEs is composed of two nested protocols, which are the protocol for VE formation phase and the protocol for VE operation phase. Figure 2 shows the interaction diagram of the proposed negotiation protocol in the form of AUML (Agent Unified Modeling Language).

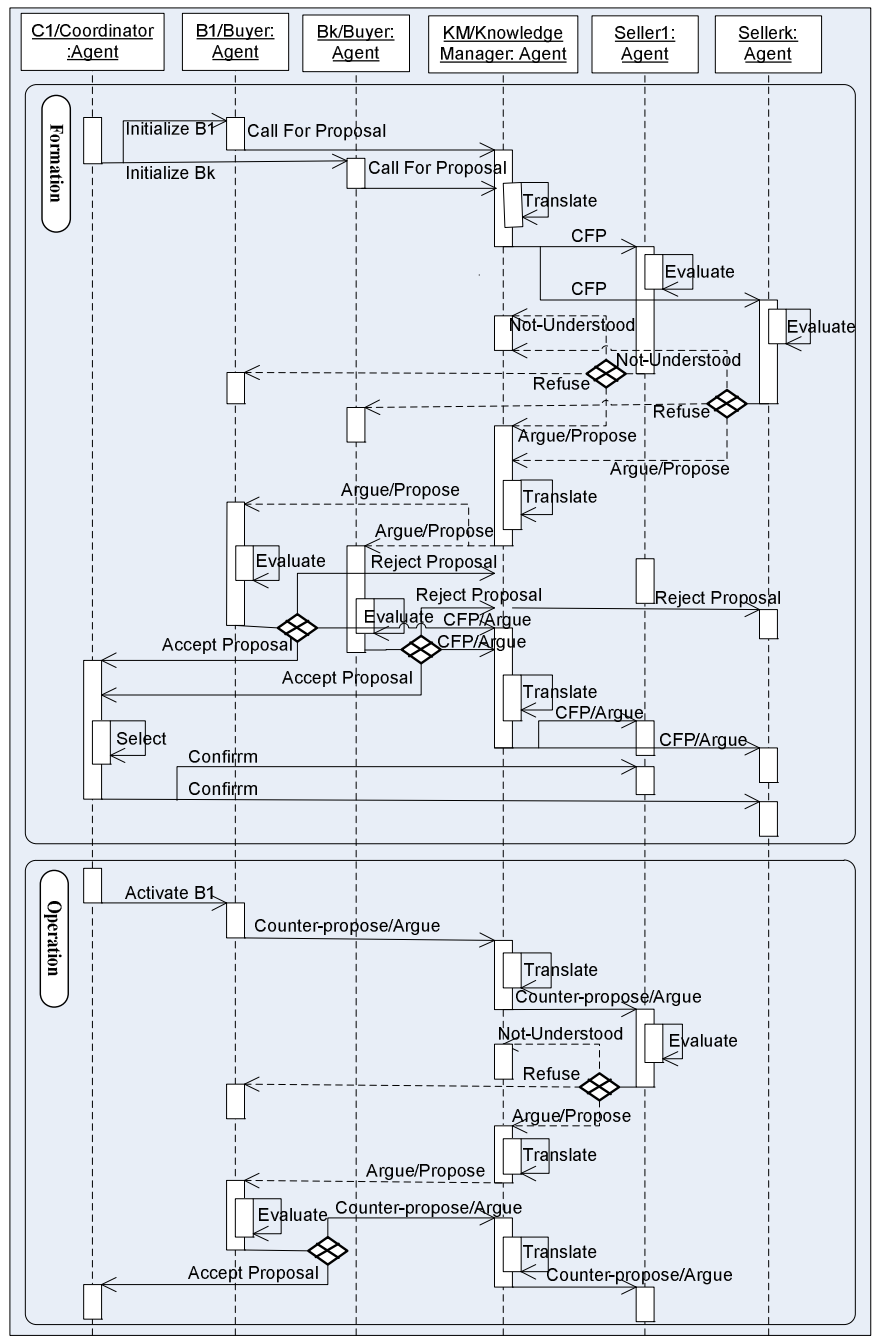

In accordance with the MAS framework, the negotiation protocol for the VE formation phase is designed to support the VE partner selection negotiation which is usually in the form of one-to-many. Extended from the CNP, three major points of adjustment are made. Firstly, a coordinator is introduced into the protocol. It initializes and governs the buyer instances to negotiate with many sellers. The one-to-many negotiation scenario can be converted to many one (buyer instance)-to-one (seller) negotiations. The overall outcomes of the multiple oneto-one negotiations are controlled by the coordinator. Secondly, the "Argue" interaction sequence is added into the interaction sequences. It encloses agents' argumentations for their current situations or against the opponent's requests. Thirdly, to support the message interoperability, a knowledge manager is also introduced into the protocol. The message formats and related terms used by different negotiation participants need to be registered within the knowledge manager. Translations are conducted through the knowledge manager to ensure negotiation counterparts' mutual understanding of messages.

In the VE operation phase, the negotiation protocol is designed for conflicts solving negotiations between VE members mainly in a one-to-one negotiation manner. If inconsistencies happen in the contract fulfillment procedure, the coordinator will activate a buyer instance which is responsible for negotiating with the contracted seller to carry out the renegotiation process. The buyer and seller can propose/counter-propose or argue to solve the inconsistency and achieve mutual agreement again. The same as in the formation phase, the interaction messages are still needed to be translated by the knowledge manager in the protocol.

For message sequences in figure 2, the CFP/Counterpropose and Propose messages are transferred between each pair of buyer agent instance and seller agent to express their negotiation issue values. If an agent identifies that it is not appropriate to propose a proposal, it can argue about its current situation using the Argue message. Agents can send NotUnderstood messages when foreign message structures or terms have been received. The Refuse message denotes the agent cannot provide the required object at the time. When an agent is content with the incoming proposal, it will send the Accept Proposal message. Otherwise, when an agent cannot make further concessions on any of the negotiation issues, it will send the Reject proposal message. The Confirm message is used to verify the mutual agreements between negotiation participants.

The major agent actions involved in the protocol are briefly introduced here. If the messages are of complex content structures (such as Argue, Propose and so on), they have to be handled through the Translate action of the knowledge manager. The Translate action involves translating message contents between agents belonging to different VE members. When a new message arrives, the buyer or seller agent evaluates the content of the message via the Evaluate action and then delivers reaction. The coordinator's Select action is for winner selection in the VE formation phase. 


\section{B. Message Structure for Negotiation}

When an agent proposes a bidding proposal or argue about its current situation, the contents of the message it sends must be adequate enough to express the entire desire of the agent. Meanwhile, the structure of the message should be refined to avoid other agents' misunderstanding. That is to say, in the protocol, the messages for negotiation should be well structured to express the involved complex information. Figure 3 shows an example of the structured message for argumentation. The message is expressed in accordance with the FIPA ACL message and content language regulation [8]. Table 1 lists the primary ACL message types and their expression meanings involved in the proposed negotiation protocol.

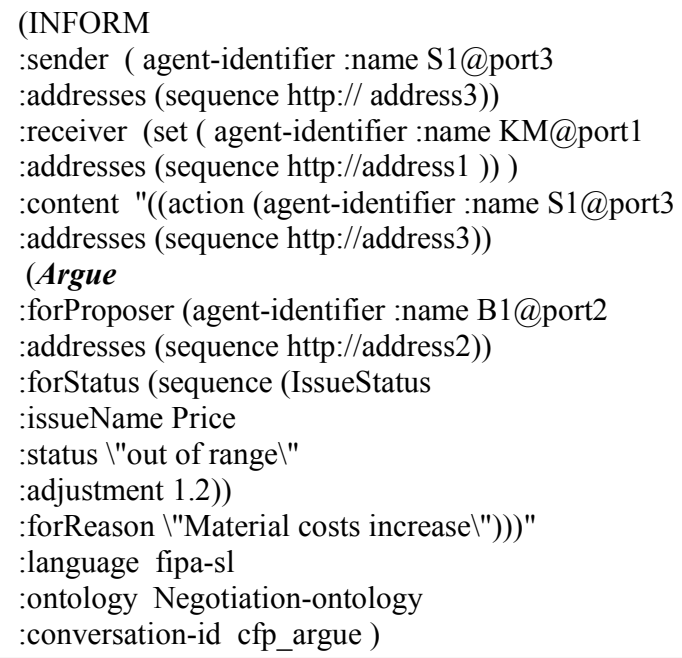

Figure 3. An INFORM message for argumentation

TABLE I. ACL Messages IN The Negotiation Protocol

\begin{tabular}{|l|l|}
\hline ACL message & Meanings in the protocol \\
\hline CFP & Call for proposal or counter-propose \\
\hline PROPOSE & Propose an offer or proposal \\
\hline INFORM & Inform or argue about the current object status \\
\hline ACCEPT-PROPOSAL & Accept a proposal or counter-proposal \\
\hline CONFIRM & Confirm the current status \\
\hline
\end{tabular}

Meanwhile, the terms used in the negotiation messages should be defined in the negotiation ontology. The negotiation ontology indicates a common vocabulary of negotiation items and some common rules of encounter [9]. Figure 4 denotes partial of the negotiation ontology defined in this paper.

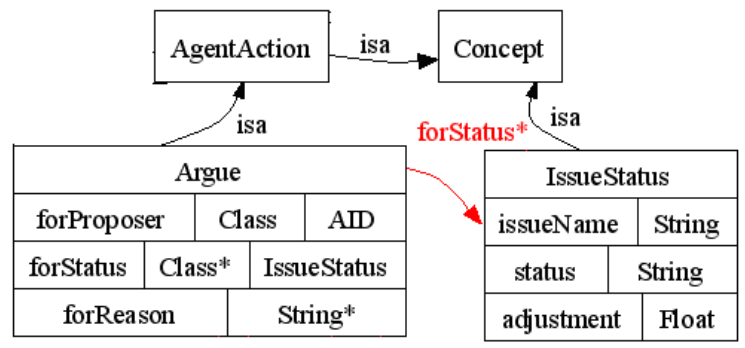

Figure 4. An INFORM message for argumentation
Here, "Argue" and "IssueStatus" are related agent action and concept to convey agents' arguments on specific negotiation issues. The agent action and concept are structured using attributes which are elicited to describe the functions and meanings of the negotiation actions and terms.

As Figure 3 denotes, the "Argue" interaction sequence in the protocol can be expressed using the ACL message "INFORM" and the "Argue" action defined in the negotiation ontology. This message presents a seller arguing about the status of price, which means, for the increase of material costs, the price requested by the buyer is out of the acceptable price range of the seller. Generally, when receiving an opponent's request (e.g. CFP), the agent checks its mental state to decide if the request goes beyond its capacity or tolerance, if so, it will give a reason for the argument and suggest a possible adjustment of the request.

\section{PRIMARY SYSTEM IMPLEMENTATION}

The proposed MAS framework and negotiation protocol will be implemented upon the JADE platform, which is a prevalent agent-oriented middleware. Assumed cases are conducted to simulate the partner selection negotiation for the formation of a VE and the subsequent contract fulfilment renegotiation for the VE operation.

\section{A. A Simulation Case for VE Formation Negotiation}

A simulation case is designed for the partner selection negotiation in the VE formation phase. In this simple case, one VE initiator (VI) is going to procure product $\mathrm{A}$ to fulfil a market opportunity. Through negotiations, the VI needs to select one partner from two potential VE partners (VP1 and VP2) to form a simple VE. VP1 and VP2 are confronting a situation of material costs increase, so their agents are prepared to argue about this situation if VI's query price is too low.

It is obvious that negotiation in this case is between one buyer and two sellers. Four types of agents are involved, which are coordinator agent (CA), knowledge manager agent (KMA), buyer agent (BA) and seller agent (SA). The CA, KMA and BA belong to the VI, while SAs represent VP1 and VP2.

Simulation experiments have been carried out to test the functioning of the system. All the agents run in the same container. CA1, KMA1, SA1 and SA2 are the local names of CA, KMA and SA. BA1 and BA2 are two instances of BA.

Firstly, order information is input into the system. Price and due date are the two negotiable issues in the order. Buyer and sellers' initial value settings of the issues are listed in table 2 .

TABLE II. InItIAL SETtings OF NegOtiation Issues

\begin{tabular}{|l|c|c|c|}
\hline & BA(BA1 and BA2) & SA1 & SA2 \\
\hline Price (HK dollars) & 168 & 280 & 270 \\
\hline Due date (days) & 46 & 63 & 60 \\
\hline
\end{tabular}

CA1 is initialized by the VI's order data, it then searches out two seller agents with local names SA1 (belonging to VP1) and SA2 (belonging toVP2) within the DF (directory facilitator). Subsequently, CA1 initializes two buyer instances BA1 and BA2 and informs them about the initial negotiation 
values. Then, BA1 and BA2 will start to negotiate with SA2 and SA1 respectively. Figure 5 shows the initial tracing results of the agent interaction sequences in VE formation phase. SAs send INFORM messages to argue that the price values in the CFP messages are too low. BA1 and BA2 then readjust the price values referring to SAs' arguments in their next CFP messages.

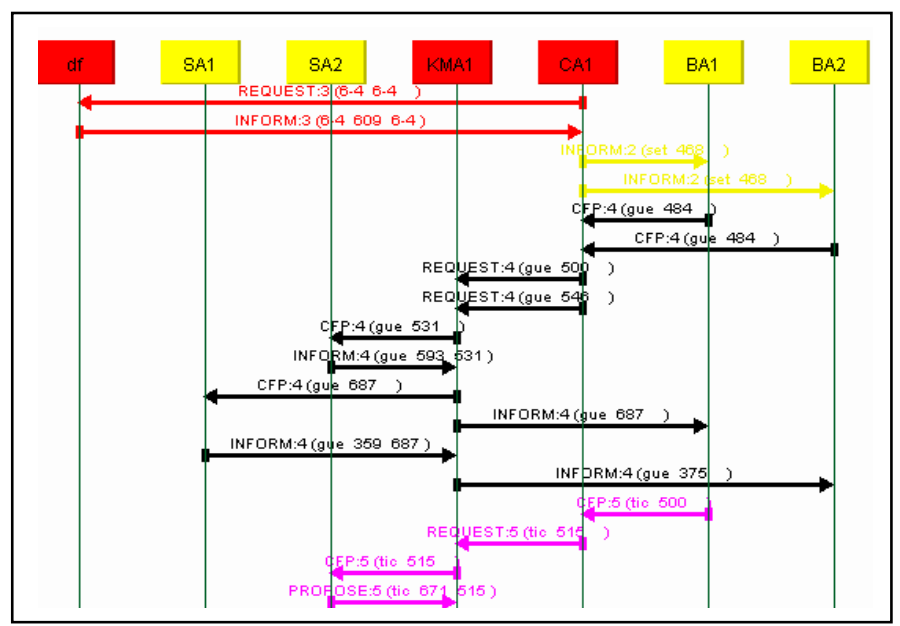

Figure 5. Agent interaction tracing diagram in VE formation phase

Table 3 shows the negotiation results in one simulation run. The SAs' argument for price is similar to the expression in Figure 3. The BA readjusts the price values accordingly. For their next CFPs, the price values increase. Finally, SA1 wins the order and is selected as the VE partner.

TABLE III. Negotiation Results IN VE Formation Phase

\begin{tabular}{|c|c|c|}
\hline & \multicolumn{2}{|c|}{ Results } \\
\hline & BA2-SA1 & BA1-SA2 \\
\hline Time limit & $\begin{aligned} \mathrm{TL}_{\mathrm{SA} 1} & =296404 \mathrm{~ms} \\
\mathrm{TL}_{\mathrm{BA} 2} & =279060 \mathrm{~ms}\end{aligned}$ & $\begin{aligned} \mathrm{TL}_{\mathrm{SA} 2} & =296388 \mathrm{~ms} \\
\mathrm{TL}_{\mathrm{BA} 1} & =279060 \mathrm{~ms}\end{aligned}$ \\
\hline $\begin{array}{llll}\text { BAs' first } & \text { CFP issue } \\
\text { values } & & & \\
\end{array}$ & $\begin{array}{c}\text { Price }_{\mathrm{BA} 2}=168 \\
\text { Duedate }_{\mathrm{BA} 2}=46\end{array}$ & $\begin{array}{c}\text { Price }_{\mathrm{BAl}}=168 \\
\text { Duedate }_{\mathrm{BAl}}=46\end{array}$ \\
\hline $\begin{array}{l}\text { BAs' adjusted issue values } \\
\text { upon SAs' argument }\end{array}$ & $\begin{array}{l}\text { Price }_{\mathrm{BA} 2}=201.86 \\
\text { Duedate } \\
\text { BA } 2=46\end{array}$ & $\begin{array}{c}\text { Price }_{\mathrm{BA} 1}=203.08 \\
\text { Duedate }_{\mathrm{BA} 1}=46\end{array}$ \\
\hline Negotiation strategies & \multicolumn{2}{|c|}{$\begin{array}{l}\text { Fixed concession rate } 0.0013 \text { or time } \\
\text { dependent concession }\end{array}$} \\
\hline Final agreement & $\begin{array}{c}\text { Price }_{\mathrm{SA} 1}=245.63 \\
\text { Duedate }_{\mathrm{SA} 1}=53\end{array}$ & $\begin{array}{l}\text { Price }_{\mathrm{SA} 2}=244.91 \\
\text { Duedate }_{\mathrm{SA} 2}=55\end{array}$ \\
\hline Negotiation rounds & $\mathrm{R}_{\mathrm{BA2}-\mathrm{SAl}}=339$ & $\mathrm{R}_{\mathrm{BA} 1-\mathrm{SA} 2}=272$ \\
\hline Buyer utility & $\mathrm{U}_{\mathrm{BA} 2-\mathrm{SAl}}=0.518$ & $\mathrm{U}_{\mathrm{BA} 1-\mathrm{SA} 2}=0.480$ \\
\hline Order winner & \multicolumn{2}{|c|}{ SA1 } \\
\hline
\end{tabular}

\section{B. A Simulation Case for VE Operation Negotiation}

The simulation case for VE operation negotiation is implemented after the VE formation negotiation has been finished. Since SA1 wins the order, VP1 is contracted as the VE partner. Then, a performance evaluator agent (PEA introduced in section 2) with local name PEA1 will be generated by CA1 to keep tracing the contract fulfillment performance of the VE partner. If the contract cannot be fulfilled, or more specifically, the ordered product cannot be delivered to the VE initiator by the end of the contract due date, PEA1 will inform CA1 to decide if renegotiation should be carried out.
In the simulation case, the contract information generated in the VE formation phase is recorded within PEA1. PEA1 will wait for the contract fulfillment information until the delivery due date of the product. To shorten the experimental time, the PEA1 will wait 104000 milliseconds instead of 53 days (which is the due date). In the experiment, the product A cannot be delivered by SA1 within the due date. Figure 6 displays a simple contract fulfillment information input GUI window. Since the contract have not been fulfilled, the due date status is null in figure 6 . Then, the PEA will send an ACL message (INFORM-IF) to inform the CA to decide if renegotiation should be carried out with the contracted seller.

\begin{tabular}{|c|c|c|c|}
\hline \multicolumn{4}{|c|}{ PEA1@828-research01:1099/JADE } \\
\hline \multicolumn{4}{|c|}{ Performance of agent: $\mathrm{SA} 1$} \\
\hline \multirow{3}{*}{$\begin{array}{l}\text { Contract ID: } \\
\text { Contract item: } \\
\text { Contract item }\end{array}$} & 001 & \multirow{2}{*}{$\begin{array}{l}\text { Product: } \\
\text { Item status: }\end{array}$} & A \\
\hline & Price & & 246 \\
\hline & Duedate & Item status & \\
\hline Deadline: & \multicolumn{2}{|c|}{ eb 04 15:24:15 CST 2010 } & Set \\
\hline \multicolumn{4}{|c|}{$\begin{array}{l}\text { Receives contract: ((action (agent-identifier :name CA1@828-research01:1099/JADE :addresses } \\
\text { Wait for } 104000 \\
\text { Performance: SA1 Price } 246 \text { Duedate null } \\
\text { PEA Sends (INFORM-IF } \\
\text { :sender (agent-identifier :name PEA1@828-research01:1099:JADE :addresses (sequence htth } \\
\text { content "((action (agent-identifier:name PEA1 @828-research01:1099/JADE :addresses (sequA } \\
\text { Ianguage fipa-sl :ontology Negotiation-ontology : conversation-id renegotiation_operation) }\end{array}$} \\
\hline \multirow[t]{2}{*}{\begin{tabular}{|l|l|l|}
4 & 111 & \\
\end{tabular}} & & & 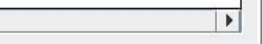 \\
\hline & & Exit & \\
\hline
\end{tabular}

Figure 6. An input GUI window for contract fulfillment information

Figure 7 shows the interaction tracing diagram of the renegotiation procedure in the VE operation phase. The negotiation messages are transferred between agents according to the negotiation protocol in the VE operation phase. CA1 activates BA2 to start renegotiation with SA1. Then, BA2 sends an INFORM message to argue about the contract item status to SA1. The following negotiation continues through exchanges of the PROPOSE and CFP messages. KMA1 still governs the translation of messages between BA1 and SA2. For example, at the beginning of renegotiation, the INFORM message is sent to SA1 through the translation of KMA1. The content structure of this message is displayed in figure 8 .

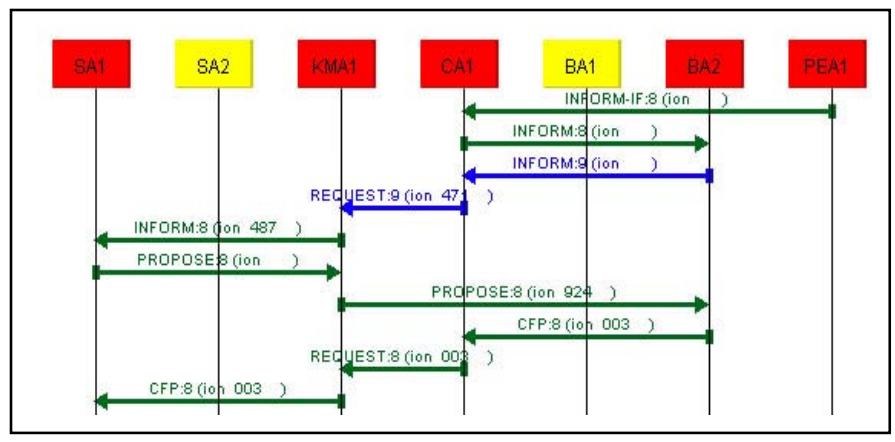

Figure 7. Agent interaction tracing diagram in VE operation phase 


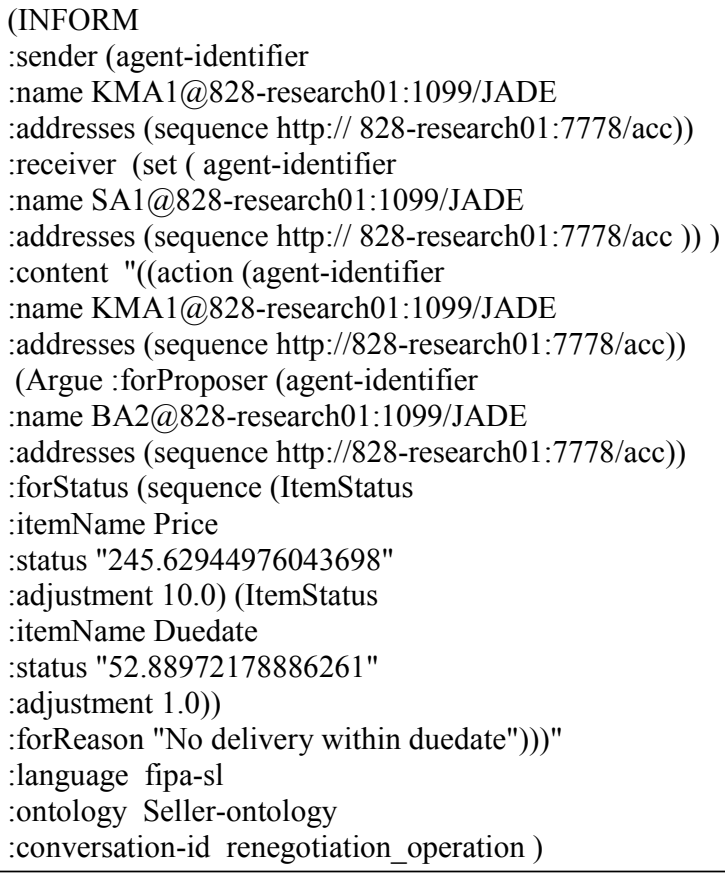

Figure 8. An ACL message translated by the KMA

After 26 rounds of negotiation, new agreement is achieved as table 4 displays. It can be seen that the delayed due date results in a lowered product price in the renegotiation procedure.

TABLE IV. RENEGOtiation RESUlts IN VE Operation PHASE

\begin{tabular}{|l|c|c|}
\hline & Price & Duedate \\
\hline Contract & 245.63 & 53 \\
\hline Renegotiation & 233.05 & 56 \\
\hline Rounds & \multicolumn{2}{|c|}{26} \\
\hline
\end{tabular}

As these two cases demonstrate, the negotiation based operation of virtual enterprises can be simulated using the proposed MAS framework. Message transmission sequences are tested in accordance with the proposed negotiation protocol for VE formation phase and VE operation phase.

\section{CONCLUSIONS AND FUTURE WORK}

This paper presents a MAS framework for the negotiation based operational integration of virtual enterprises. In the framework, six types of intelligent agents have been designed to perform different functional activities in the VE life cycle. A two-phase negotiation protocol supporting agent argumentation has been developed to handle the partner selection and conflict solving negotiation scenarios in the $\mathrm{VE}$ formation and operation phases. In the current stage, the VE formation phase and operation phase of the proposed framework have been implemented upon the JADE platform. The primary system simulation results show that agents can interact smoothly under the proposed negotiation protocol.

For future work, more simulation tests and comparison experiments in various negotiation situations will be designed to further evaluate the effectiveness and efficiency of the system architecture and negotiation protocol.

\section{REFERENCES}

[1] L. M. Camarinha-Matos, H. Afsarmanesh, C. Garita, and C. Lima, "Towards an architecture for virtual enterprises " Journal of Intelligent Manufacturing, vol. 9, pp. 189-199, 1998.

[2] Y. E. Nahm and H. Ishikawa, "A hybrid multi-agent system architecture for enterprise integration using computer networks," Robotics and Computer-Integrated Manufacturing, vol. 21, pp. 217-234, 2005.

[3] K. H. Choi, D. S. Kim, and Y. H. Doh, "Multi-agent-based task assignment system for virtual enterprises," Robotics and ComputerIntegrated Manufacturing, vol. 23, pp. 624-629, 2007.

[4] L. Mikhailov, "Fuzzy analytical approach to partnership selection in formation of virtual enterprises," The International Journal of Management Science, vol. 30, pp. 393-401, 2002.

[5] T. Y. Kim, S. Lee, K. Kim, and C. H. Kim, "A modeling framework for agile and interoperable virtual enterprises," Computers in Industry, vol. 57, pp. 204-217, 2006.

[6] P. Faratin, C. Sierra, and N. R. Jennings, "Negotiation decision functions for autonomous agents," International Journal of Robotics and Autonomous Systems, vol. 24, pp. 159-182, 1998.

[7] R. Smith, "The contract net protocol: high-level communication and control in a distributed problem solver," IEEE Transactions on Computers, vol. 29, pp. 1104-1113, 1980.

[8] FIPA, Foundation for Intelligent Physical Agents, http://www.fipa.org

[9] V. Tamma, S. Phelps, I. Dickinson, and M. Wooldridge, "Ontologies for supporting negotiation in e-commerce," Engineering Applications of Artificial Intelligence, vol. 18, pp. 223-236, 2005. 